\title{
Mamíferos medianos y grandes del Espacio Territorial de Capacitación y Reincorporación (ETCR) Playa Rica, La Macarena, Meta, Colombia
}

\author{
Carlos A. Aya-Cuero1 (iD), Camila A. Díaz-B² (D), Diego A. Esquivel ${ }^{1,3 *}$ (iD \\ 1 Fundación Kurupira, Bogotá, Colombia. \\ 2 Grupo de conservación y manejo de vida silvestre, Universidad Nacional de Colombia, Bogotá, Colombia. \\ 3 Bird and Mammal Evolution, Systematics and Ecology Lab, Universidade Federal do Rio Grande do Sul, Porto Alegre-RS, \\ Brasil. \\ *Correspondencia: diegodaem@gmail.com
}

\begin{abstract}
Resumen
Las sabanas del Yari son una importante área biogeográfica cuya biodiversidad no ha sido explorada. Esta zona estuvo históricamente bajo el control de los grupos armados ilegales que restringían su acceso e investigación. Con el proceso de paz, las sabanas del Yarí se abrieron a la investigación, pero también a procesos antrópicos que amenazan su biodiversidad. Aquí, presentamos un listado preliminar que incluye los primeros registros de mamíferos medianos y grandes del sector noroccidental de las sabanas del Yarí, como resultado de un programa de monitoreo comunitario con excombatientes de las FARC. Reportamos 24 especies pertenecientes a 18 familias y 8 órdenes de mamíferos. Resaltamos la presencia de diez especies amenazadas incluidas Priodontes maximus, Tapirus terrestris y Ateles belzebuth. Esto representa el $96 \%$ de los mamíferos terrestres en el río Yarí. A partir de las entrevistas semiestructuradas y notas de campo consideramos a la deforestación, el avance de la frontera agrícola, la ganadería y la caza como las principales amenazas para los mamíferos.
\end{abstract}

Palabras clave: cámaras trampa, excombatientes, Llanos orientales, sabanas naturales.

Abstract

The Yari savannas are an important biogeographic area whose biodiversity has not been explored. This area was historically under the control of illegal armed groups who restricted their access and investigation. With the peace process, the Yari savannas were opened to research, but also to anthropic processes that threaten their biodiversity. Here, we present a preliminary list that includes the first records of medium and large mammals from the northwestern sector of the Yari savannas, as a result of a community monitoring program with former FARC combatants. We report 24 species belonging to 18 families and 8 orders of mammals. We highlight the presence of ten threatened species including Priodontes maximus, Tapirus terrestris and Ateles belzebuth. This represents $96 \%$ of the terrestrial mammals in the Yari River. From the semi-structured interviews and field notes, we consider deforestation, advance of the agricultural frontier, cattle ranching and hunting as the main threats to mammals.

Key words: camera traps, eastern plains, excombatants, natural savannas. 
Las sabanas naturales en Colombia tienen una extensión aproximada de 23 millones de hectáreas, que constituyen un 20 \% de Colombia (Rippstein et al. 2001). Dentro de estas se encuentran las sabanas del Yarí, las cuales hacen parte de la Altillanura de la Orinoquia y limitan con la selva amazónica (Botero \& Serrano 2019). Se encuentran ubicadas al sur de la serranía de La Macarena, entre los departamentos de Caquetá, Guaviare y Meta. Desde una perspectiva biogeográfica, esta zona es de gran interés, ya que hace parte del corredor biológico que conecta la amazonia con la región andina (Botero \& Serrano 2019), por lo que su fauna y flora está conformada por elementos andino-amazónicos. Recientemente el sector nororiental de estas sabanas fue incluido como parte de las nuevas áreas del Parque Nacional Natural (PNN) Serranía de Chiribiquete (Rojas et al. 2019).

Las sabanas del Yarí son una zona desconocida, ya que históricamente fue ocupada por grupos armados ilegales, que impedían su acceso e investigación. Después del proceso de paz los excombatientes se encuentran en 24 Espacios Territoriales de Capacitación y Reincorporación (ETCR), distribuidos en 13 departamentos del país (ARN 2019). Con la conformación del ETCR Playa Rica, los excombatientes han dejado las sabanas del Yarí abiertas a futuras investigaciones, pero lamentablemente, también al aumento de actividades como la deforestación, colonización y extensión de la frontera agrícola y ganadera (Romero-Ruiz et al. 2011).

De acuerdo con Bertassoni et al. (2019) los cambios en el uso del suelo tienen complejas características políticas y socioeconómicas que generan impactos directos sobre la biodiversidad, como la pérdida y degradación de los ecosistemas y la disminución poblacional de mamíferos medianos y grandes. En este sentido, los inventarios de especies son una herramienta fundamental para el diseño de estrategias apropiadas de conservación, la investigación de procesos macro ecológicos y el estudio funcional de los ecosistemas (Boitani et al. 2011). En este estudio presentamos un inventario preliminar de los mamíferos medianos y grandes del sector noroccidental de las sabanas del Yarí, departamento del Meta, como resultado de una estrategia de monitoreo comunitario con excombatientes de las FARC, en el cual 25 personas realizaron actividades muestreo de mamíferos como parte de su proyecto transversal de educación secundaria. En el siguiente video se documenta esta iniciativa https://www.youtube.com/watch?v=d3YvMump60\&t=17s

El ETCR Playa Rica (2,053863, -74,254903; WGS84), está localizado al suroccidente del Departamento del Meta, en el municipio de La Macarena, a una elevación de 250 msnm. Esta zona presenta una temperatura promedio de $25^{\circ} \mathrm{C}$, una precipitación media anual de $2.914 \mathrm{~mm}$ y una humedad relativa del 86 \%. (Lasso et al. 2018; Figura 1).

Con el objetivo de registrar los mamíferos silvestres medianos y grandes presentes en esta área, realizamos un muestreo durante seis meses continuos entre julio y diciembre del año 2017 (final de la época de lluvias e inicio de la época seca). En este periodo instalamos seis cámaras trampa (Bushnell Trophy Cam) en ocho estaciones de muestreo, las cuales fueron programadas para registrar tanto videos como fotografias durante las 24 horas del día. Adicionalmente, realizamos recorridos diurnos (6:00 - 8:00 am) y nocturnos (6:00 - 8:00 pm) de $2 \mathrm{~km}$ en promedio por día durante 25 días y entrevistas semiestructuradas sobre diversidad y uso de la fauna a los excombatientes que recorrían estas áreas, así como a campesinos y cazadores de la zona. Se anotaron y fotografiaron evidencias indirectas como las huellas encontradas en los recorridos. Para la 
identificación de las especies usamos las claves de Emmons \& Feer (1997) y Linares (1999), para actualizar la taxonomía se siguió a Ramírez-Chaves et al. (2019). Las categorías de amenaza se consultaron a partir de la lista roja de la UICN (2020) y su inclusión en los apéndices de la CITES (2020). Para analizar los registros consideramos como eventos independientes aquellos registros (videos o fotografías) con más de tres horas de diferencia entre ellos.
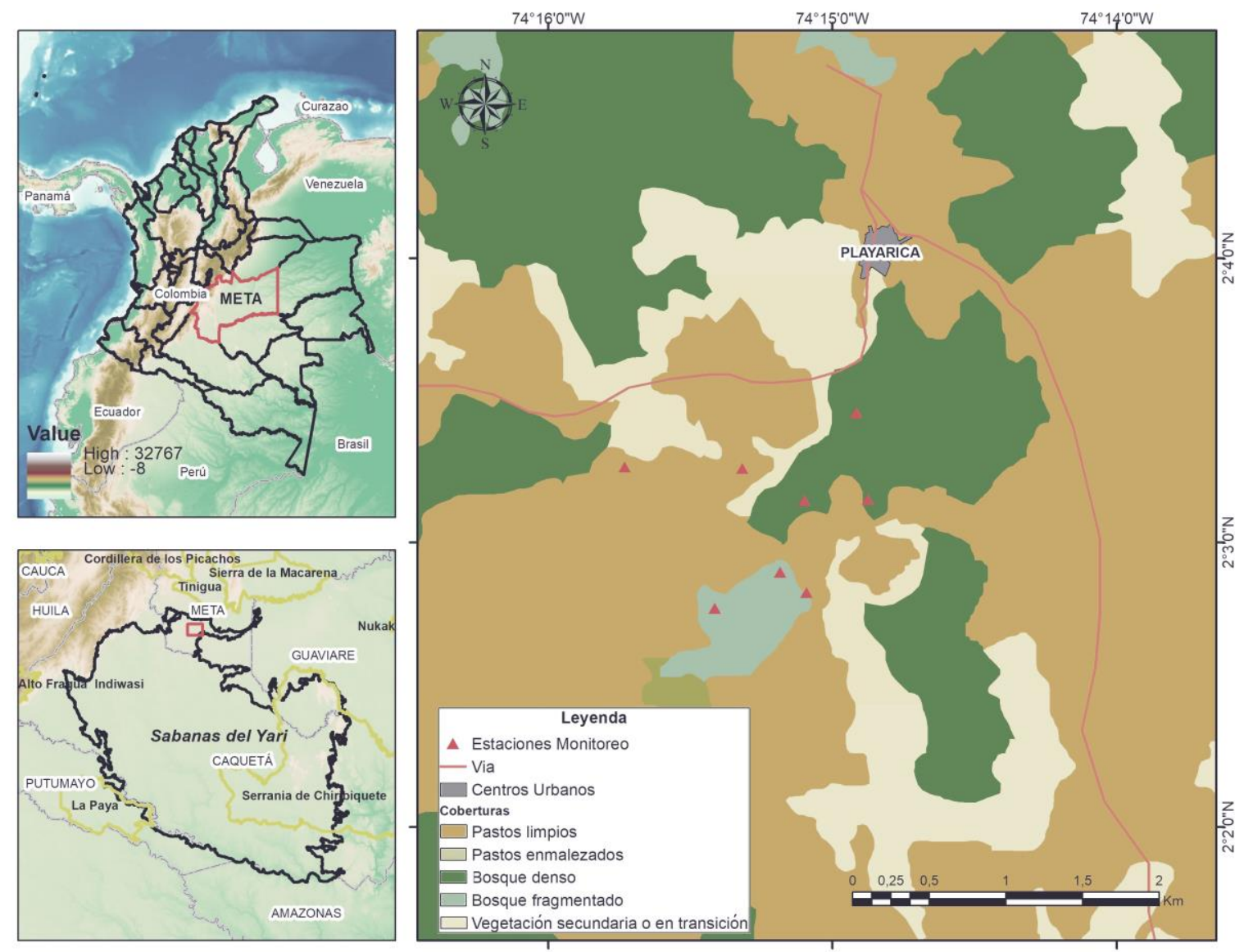

FIGURA 1. Ubicación geográfica del ETCR Playa Rica y estaciones de muestreo con cámaras trampa.

Con un esfuerzo de muestreo de 540 noches/cámara y $50 \mathrm{~km}$ recorridos registramos un total de 8 órdenes, 18 familias y 24 especies de mamíferos medianos y grandes (Tabla 1). A partir del fototrampeo fue posible el registro de 17 de estas especies. Resaltamos la presencia de diez especies amenazadas entre las que se destacan Ateles belzebuth (Atelidae, Primates) categorizada En Peligro, Priodontes maximus (Chlamyphoridae, Cingulata) considerada Vulnerable y categorizada en el apéndice I de CITES (2020) y Tapirus terrestris (Tapiridae, Perissodactyla) en categoría Vulnerable (VU, UICN 2020, Figura 2).

Estos registros son de especial interés al considerar las pocas investigaciones que se han realizado en las sabanas del Yarí, dadas las dificultades de orden público que se han presentado históricamente en la zona (Espinoza et al. 2012). Esta falta de investigaciones ha causado una carencia de información científica que junto a la pérdida de cobertura vegetal son las principales amenazas para las poblaciones de mamíferos. En campo se 
evidenció que algunas de las especies registradas se enfrentan a diferentes presiones que incluyen su uso como mascota (T. terrestris, C. paca, L. lagotricha, P. tajacu), caza por retaliación (C. thous, L. longicaudis, P. concolor) y caza por subsistencia (C. paca, D. pastasae y D. novemcinctus).

TABLA 1. Mamíferos medianos y grandes registrados en el ETCR Playa Rica, sector noroccidental de las Sabanas del Yarí. Tipo de registro: (CT: cámara trampa, E: entrevistas, El: evidencia indirecta, O: observación directa).

\begin{tabular}{|c|c|c|c|c|c|c|c|c|}
\hline \multirow[b]{2}{*}{ Familia } & \multirow[b]{2}{*}{ Especie } & \multirow{2}{*}{ Nombre común } & \multicolumn{4}{|c|}{ Tipo de registro } & \multirow{2}{*}{ CITES } & \multirow{2}{*}{ UICI } \\
\hline & & & CT & E & $\mathrm{El}$ & 0 & & \\
\hline Didelphidae & Didelphis marsupialis & Chucha, zarigüeya & $x$ & & & & & LC \\
\hline Myrmecophagidae & Tamandua tetradactyla & Oso melero & $x$ & & & & & LC \\
\hline \multirow[t]{2}{*}{ Dasypodidae } & Dasypus novemcinctus & $\begin{array}{l}\text { Armadillo nueve } \\
\text { bandas }\end{array}$ & $x$ & $x$ & & $x$ & & LC \\
\hline & Dasypus pastasae & Armadillo espuelón & $x$ & & & $x$ & & NE \\
\hline Chlamyphoridae & Priodontes maximus & Ocarro & $x$ & & & & I & VU \\
\hline \multirow[t]{2}{*}{ Felidae } & Leopardus pardalis & Ocelote & $x$ & & & & I & LC \\
\hline & Puma concolor & Puma & & $x$ & & $x$ & II & LC \\
\hline Canidae & Cerdocyon thous & Zorro perro & $x$ & & & $x$ & II & LC \\
\hline \multirow[t]{2}{*}{ Mustelidae } & Eira barbara & Taira & $x$ & & & & III & LC \\
\hline & Lontra longicaudis & Nutria & & $x$ & & $x$ & 1 & NT \\
\hline \multirow[t]{2}{*}{ Procyonidae } & Nasua nasua & Coatí & $x$ & & & & III & LC \\
\hline & Procyon cancrivorus & Mapache & $x$ & & $x$ & & & LC \\
\hline Tapiridae & Tapirus terrestris & Tapir, danta & $x$ & & $x$ & $x$ & ॥ & VU \\
\hline Tayassuidae & Pecari tajacu & Pecarí de collar & $x$ & & & $x$ & II & LC \\
\hline Cervidae & Odocoileus cariacou & Ciervo & $x$ & & & & III & NE \\
\hline \multirow[t]{3}{*}{ Atelidae } & Alouatta seniculus & Mono aullador & & $x$ & $x$ & $x$ & ॥ & LC \\
\hline & Ateles belzebuth & Mono araña & & & & $x$ & II & EN \\
\hline & Lagothrix lagotricha & Mono lanudo & & & & $x$ & ॥ & VU \\
\hline Callitrichidae & Leontocebus nigricollis & Tamarino & & & & $x$ & & LC \\
\hline Cebidae & Sapajus apella & Mono maicero & $x$ & & & & & LC \\
\hline Pitheciidae & Cheracebus lugens & Socayo negro & & & & $x$ & & LC \\
\hline Erethizontidae & Coendou prehensilis & Puercoespín & & $x$ & & $x$ & & LC \\
\hline Cuniculidae & Cuniculus paca & Guagua & $x$ & & & $x$ & III & LC \\
\hline Dasyproctidae & Dasyprocta fuliginosa & Picure & $x$ & & & $x$ & III & LC \\
\hline
\end{tabular}

El número de especies registrados en este trabajo corresponde al $96 \%$ de los mamíferos medianos y grandes registradas por Atuesta-Dimian \& Ganeden (2019) para la cuenca alta del río Yarí, una de las localidades más cercanas con estudios disponibles. No obstante, reportamos siete especies adicionales que no fueron encontradas por dichos autores. Asimismo, a pesar de la diferencia entre los esfuerzos de muestreo, utilizando cámaras trampa se logró registrar un número de especies comparable con otras zonas de estudio bien conservadas como Caño Cristales (21 especies a partir de 742 noches/trampa), la cuenca alta del río Yarí (17 especies a partir de 244 noches/trampa) y la cuenca media del río Planas (24 especies a partir de 1789 noches/trampa), estudios descritos por Díaz-Pulido et al. (2017), Atuesta-Dimian \& Ganeden (2019) y Aya-Cuero et al. (2019) respectivamente. 

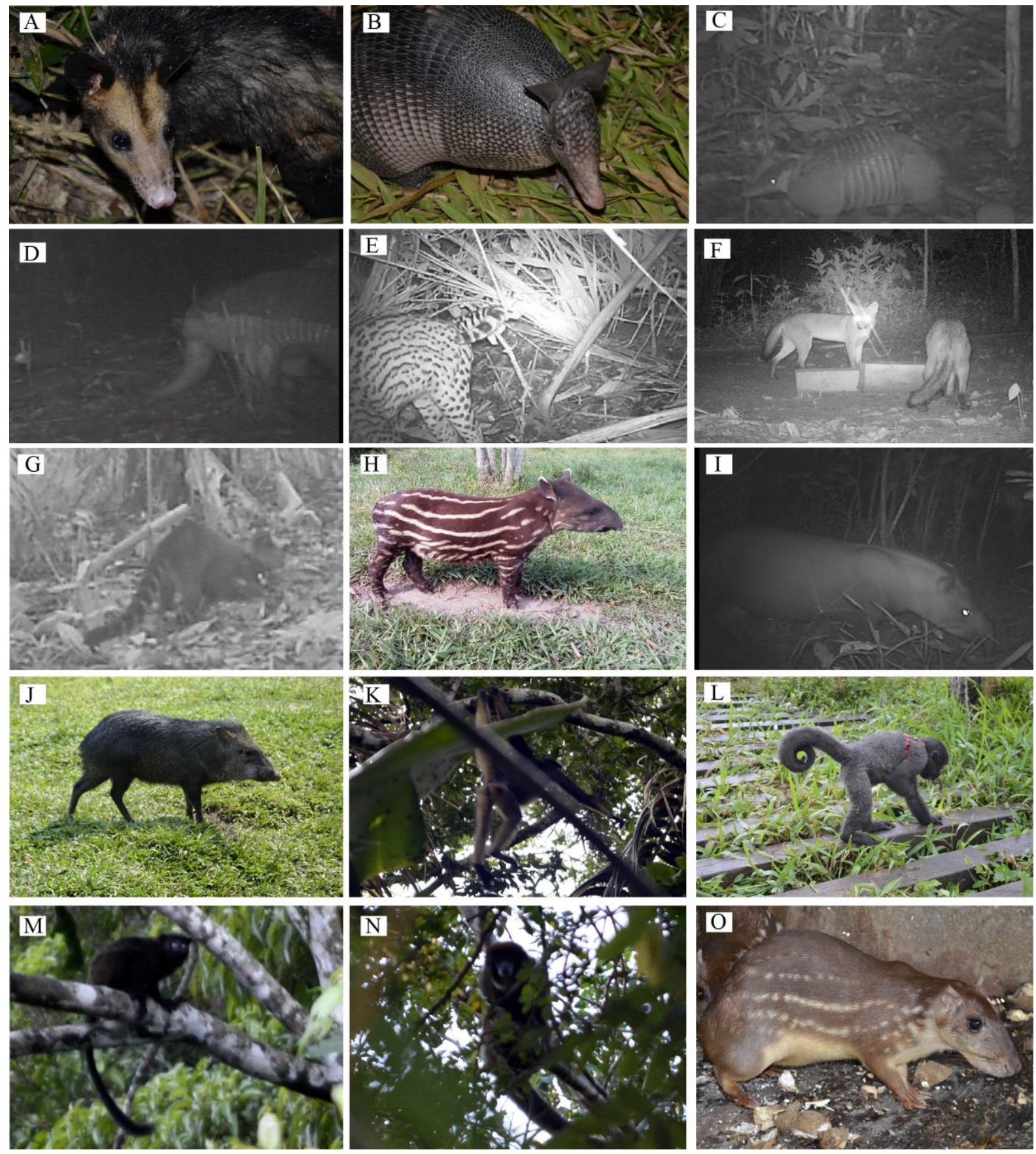

FIGURA 2. Algunas especies de mamíferos registradas en el ETCR Playa Rica, La Macarena, Meta, Colombia. A. Didelphis marsupialis, B. Dasypus novemcinctus, C. Dasypus pastasae, D. Priodontes maximus, E. Leopardus pardalis, F. Cerdocyon thous, G. Nasua nasua, H y I. Tapirus terrestris J. Percari tajacu, K. Ateles belzebuth, L. Lagotrix lagotricha, M. Leontocebus nigricollis, N. Cheracebus lugens.. O. Cuniculus paca.

Los listados de especies permiten conocer la diversidad de un lugar determinado, y también para la toma decisiones de conservación (Solari et al. 2013). Esto es especialmente importante en áreas que son transformadas rápidamente y que son habitadas repentinamente como ocurre con los ETCR que se conformaron en todas las 
regiones biogeográficas de Colombia. En ese sentido, este estudio constituye uno de los primeros trabajos que describe la diversidad de mamíferos en un ETCR en Colombia, y proporciona una base para investigar el impacto de estos espacios sobre los mamíferos a largo plazo.

Resaltamos la importancia de continuar con los esfuerzos para documentar la diversidad de mamíferos en las sabanas del Yarí, especialmente en los grupos de micromamíferos terrestres y voladores, con los cuales se complementarían los datos e información relevante para desarrollar estrategias de conservación en esta zona. Asimismo, es importante reforzar estrategias de educación ambiental con los pobladores de la zona, tanto excombatientes como comunidad aledaña, a fin de favorecer la conservación de especies cuyas poblaciones se encuentran altamente amenazadas.

\section{AGRADECIMIENTOS}

Agradecemos a los directivos del ETCR Playa Rica, a los excombatientes e integrantes del Grupo de Monitoreo de Fauna y a Keiler Tellez por su constante apoyo en campo. A la Fundación Omacha por el préstamo de equipos de campo y a Cristian Moreno por su apoyo en la elaboración del mapa del área de estudio.

\section{REFERENCIAS}

Agencia Para la Reincorporación y Normalización (ARN). 2019. Espacios Territoriales de Capacitación y Reincorporación. www.reincorporacion.gov.co. Consultada el 1 de Mayo de 2020.

Atuesta-Dimian N, Ganeden J. 2019. Mamíferos medianos y grandes de las cuencas altas de los ríos Tunia, Itilla y Yarí. En: Fundación para la Conservación y Desarrollo Sostenible. Expediciones científicas en las áreas del PNN Serranía del Chiribiquete. Bogotá, D.C., Colombia. p. 77-93.

Aya-Cuero CA, Mosquera-Guerra F, Esquivel DA, Trujillo F, Brooks D. 2019. Medium and large mammals of the mid Planas River basin, Colombia. Biota Colombiana 20:76-92. https:// doi.org/10.210068/c2019.v20n02a06

Bertassoni A, Costa RT, Gouvea JA, Bianchi RDC, Ribeiro JW, Vancine MH, Ribeiro MC. 2019. Land-use changes and the expansion of biofuel crops threaten the giant anteater in southeastern Brazil. Journal of Mammalogy 100:435-444. https:// doi.org/10.1093/jmammal/gyz042

Boitani L, et al. 2011. What spatial data do we need to develop global mammal conservation strategies? Philosophical Transactions of the Royal Society B: Biological Sciences 366:26232632. https://doi.org/10.1098/rstb.2011.0117

Botero P, Serrano H. 2019. Caracterización Biofísica del área de ampliación, geología, geomorfología y suelos. En: Fundación para la Conservación y Desarrollo Sostenible. Expediciones científicas en las áreas del PNN Serranía del Chiribiquete. Bogotá, D.C., Colombia. p. 21-30.

CITES. 2020. Convention on International Trade in Endangered Species of Wild Fauna and Flora. https:// cites.org/. Consultada el 23 de Agosto de 2020.

Díaz-Pulido A, Velásquez T, López A, Alfonso J, Mantilla-Meluk H. 2017. Mamíferos. En: Lasso CA, Morales-B MA (Eds). III Fauna de Caño Cristales, Sierra de La Macarena, Meta, Colombia. 
Serie Editorial Fauna Silvestre Neotropical. Instituto de Investigación de Recursos Biológicos Alexander von Humboldt (IAvH). Bogotá, D. C., Colombia. p. 157-183.

Emmons L, Feer F. 1997. Neotropical rainforest mammals: a field guide. Chicago, USA: University of Chicago Press.

Espinosa N, González E, Ramírez E. 2012. Etnografia, territorio y conflicto armado. Metodología de una investigación sobre la construcción regional de los llanos del Yarí (Caquetá, Colombia). El Ágora 12:329-348.

Lasso CA, Morales-Betancourt MA, Escobar-Martínez ID. 2018. V. Biodiversidad de la sierra de La Macarena, Meta, Colombia. Parte I. Ríos Guayabero medio, bajo Losada y bajo Duda. Serie Editorial Fauna Silvestre Neotropical. Instituto de Investigación de Recursos Biológicos Alexander von Humboldt. Bogotá, D.C., Colombia. p. 329.

Linares O. 1998. Mamíferos de Venezuela. Sociedad Conservacionista AUDUBON de Venezuela, Caracas, Venezuela, p. 691

Ramírez-Chaves HE, Suárez-Castro AF, Sociedad Colombiana de Mastozoología, Zurc D, Concha Osbahr DC, Trujillo A, Noguera-Urbano EA, Pantoja GE, Rodríguez-Posada ME, GonzálezMaya JF, Pérez-Torres J, Mantilla-Meluk H, López-Castañeda C, Velásquez-Valencia A, Zárrate-Charry D. 2019. Mamíferos de Colombia. Versión 1.6. Sociedad Colombiana de Mastozoología. Consultada el 10 de Agosto de 2020. Checklist dataset https://doi.org/10.15472/kl1whs

Rippstein G, et al. 2001. Agroecología y biodiversidad de las sabanas en los Llanos Orientales de Colombia. Centro Internacional de Agricultura tropical. Cali, Colombia.

Rojas et al. 2019. Expediciones científicas en las nuevas áreas del PNN Serranía de Chiribiquete. Bogotá, D.C., Colombia: Fundación para la Conservación y el Desarrollo Sostenible.

Romero-Ruiz M, et al. 2011. Landscape transformations in savannas of northern South America: Land use/ cover changes since 1987 in the Llanos Orientales of Colombia. Applied Geography 32:766-776.

Solari S, Muñoz-Saba Y, Rodríguez-Mahecha JV, Defler T, Ramírez-Chaves H. Trujillo F. 2013. Diversidad, Endemismo y Conservación de los Mamíferos de Colombia. Mastozoología Neotropical 20:301-365.

UICN. 2020. The IUCN Red List of Threatened Species. https:/ /www.iucnredlist.org. Consultado el 21 de Agosto de 2020

Editor: Diego J. Lizcano

Recibido 2020-08-30

Revisado: 2020-09-05

Aceptado 2020-09-07

Publicado 2020-12-21 